\title{
Associations Between Variations in Breast Anatomy and Early Breastfeeding Challenges
}

\section{Alison K. Ventura, PhD, CLEC' ${ }^{1}$, Brittany Lore, BS ${ }^{\prime}$, and Olga Mireles, RN, IBCLC²}

\begin{abstract}
Background: Mothers with anatomic variability (e.g., shorter, wider nipples; denser areolas) may experience breastfeeding challenges disproportionately.

Research aim: To examine whether variations in breast anatomy are associated with risk for early breastfeeding challenges.

Methods: Participants included mothers < 6 weeks postpartum. Nipple base width, nipple length, and areolar density were measured on the right and left breast separately. Experiences with early breastfeeding challenges were determined through a combination of maternal report and clinical assessment.

Results: Participants $(N=119)$ had an average nipple diameter of $23.4(S D=3.0) \mathrm{mm}$ for left nipples and $23.5(S D=3.0)$ $\mathrm{mm}$ for right nipples (range $=10-34 \mathrm{~mm}$ ). Average nipple length was $8.5(S D=3.2) \mathrm{mm}$ for left breasts and 9.1 $(S D=3.2)$ $\mathrm{mm}$ for right breasts (range $=5-20 \mathrm{~mm}$ ); 35\% of participants had dense areolas on the left breast and $36 \%$ had dense areolas on the right breast. The combination of wider and longer nipples was associated with greater risk for difficulties with latch; the combination of wider nipples and denser areolas was associated with greater risk for sore nipples. For participants with more dense areolas, shorter and wider nipples were associated with greater risk for low milk supply and slow infant weight gain. For participants with less dense areolas, longer and wider nipples were associated with greater risk for low milk supply and slow infant weight gain.
\end{abstract}

Conclusion: Further research is needed to understand how measures of breast anatomy can be used to guide targeted intervention efforts.

\section{Keywords}

anatomy, breast, breastfeeding, breastfeeding difficulties, latch-on, milk supply

Despite the unparalleled benefits of breastfeeding for infants, mothers, and families, only $13 \%$ of the population in the United States meets the American Academy of Pediatrics breastfeeding recommendations (American Academy of Pediatrics , 2012). Unsuccessful initiation of breastfeeding is a primary barrier to continued breastfeeding, and up to $40 \%$ of mothers experience early breastfeeding challenges, including difficulty establishing an effective latch and experiences with sore, wounded, and/or cracked nipples (Feenstra et al., 2018). Understanding the reasons for early breastfeeding challenges is important for highlighting targets for early prevention and intervention efforts.

Through clinical observations, Wilson-Clay and Hoover (2017) speculated that mothers with anatomic variability (e.g., relatively larger breasts, shorter and wider nipples, and denser areolas) disproportionately experienced early breastfeeding challenges. Early breastfeeding challenges may be due, in part, to disparities between mothers' breast anatomy and infant oral anatomy and sucking abilities. Although it is likely that over time and as the infant

\footnotetext{
'Department of Kinesiology and Public Health; Center for Health Research, California Polytechnic State University, San Luis Obispo, CA, USA

${ }^{2}$ Dignity Health French Hospital Medical Center Breastfeeding Center, San Luis Obispo, CA, USA
}

\section{Corresponding Author:}

Alison K. Ventura, PhD, CLEC, California Polytechnic State University, Department of Kinesiology and Public Health, Center for Health Research, One Grand Ave, San Luis Obispo, CA 93407, USA.

Email: akventur@calpoly.edu 


\section{Key Messages}

- Few researchers have studied associations between mothers' anatomical breast variations and risk for early breastfeeding challenges.

- Varying combinations of nipple width, nipple length, and areolar density were associated with most of the breastfeeding challenges examined.

- Mothers with variations in breast anatomy may need anticipatory guidance and targeted interventions to overcome early breastfeeding challenges.

matures, the infant's oral cavity will eventually grow to accommodate the size of the breast, nipples, and areolas, it is possible that this initial disparity between maternal and infant anatomy may cause early breastfeeding challenges and impede the establishment of breastfeeding during early infancy (Wilson-Clay \& Hoover, 2017). Although Wilson-Clay and Hoover (2017) posit larger breasts, shorter and wider nipples, and denser areolas as a possible source of early difficulties with infant latch and related sequelae, few investigators have empirically assessed this possibility. In one of the only available studies on this topic, Vazirinejad et al. (2009) found that mothers' anatomic breast variations (defined as relatively large, flat, or inverted nipples and/or relatively large breasts) were associated with poorer weight gain for neonates. In two additional studies, nipple lengths of at least $7 \mathrm{~mm}$ were associated with successful initiation of breastfeeding (Puapornpong et al., 2013), whereas smaller breast size and longer nipple lengths were associated with longer breastfeeding durations (Mangel et al., 2019; Puapornpong et al., 2013).

The aim of the present study was to address this research gap by examining whether variations in breast anatomy are associated with risk for early breastfeeding challenges, including poor latch, sore and cracked nipples, low milk supply, and/or slow infant weight gain. The ultimate goal of this study was to provide health professionals with empirical data to support evidence-based, targeted interventions. It was hypothesized that women assessed to have variations in breast anatomy characterized by shorter and wider nipples and denser areolas would be at a higher risk for difficulties with latch, sore and cracked nipples, mastitis, low milk supply, and/or slow infant weight gain.

\section{Methods}

\section{Design}

This was a cross-sectional, observational study (Bordens \& Abbot, 2018). This research design was appropriate given that the aim of this study was to examine associations between exposures (variations in nipple anatomy) and multiple outcomes (breastfeeding challenges) measured simultaneously (Tabachnick \& Fidell, 2019). The California Polytechnic State University Institutional Review Board and the Dignity Health Northridge Institutional Review Board approved all study procedures.

\section{Setting}

All assessments were conducted within a private exam room at The Breastfeeding Center at the French Hospital Medical Center (FHMC) in San Luis Obispo, CA. FHMC serves San Luis Obispo County, which is located on the Central Coast of California. At the time of the present study, the population of San Luis Obispo County was $\sim 284,000$. The racial and ethnic composition of residents was: $69 \%$ non-Hispanic/Latino/Latinx white; $23 \%$ Hispanic/Latino/Latinx; 3\% Asian; 2\% black; and 1\% American Indian or Alaska Native. Median household income was $\sim \$ 71,000$. FHMC was designated a BabyFriendly Hospital by Baby-Friendly USA and had a 99\% in-hospital breastfeeding rate. Per hospital policy at the time of the present study, all mothers who gave birth at FHMC were provided with access to the lactation support providers in the FHMC Breastfeeding Center and were seen for both preventive counseling and help with breastfeeding challenges.

\section{Sample}

The study's target population was mothers with infants 6 weeks of age or younger who visited the FHMC Breastfeeding Center for any reason (e.g., preventive counseling, lactation support). Mothers were eligible if they were: (1) 18 years of age or older; (2) had a singleton infant born full-term ( $>37$ weeks' gestation) who was between birth and 6 weeks of age at the time of the consultation; and (3) spoke either English or Spanish. Mothers were ineligible if they: (1) did not meet that aforementioned inclusion criteria; (2) had a medical history of breast surgery (e.g., augmentation, reduction, biopsy); (3) had an infant with a medical condition that interfered with feeding; or (4) had an infant taking a medication of any type.

A convenience sampling method was used; all mothers who visited the FHMC Breastfeeding Center and who met the eligibility criteria were invited to participate in the study. A total of 119 participants provided full data on the exposures (i.e., variation in nipple anatomy) and outcomes (i.e., breastfeeding challenges) of interest. The analytic sample for all inferential analyses was $n=115$ because four participants were missing data for included covariates: three participants did not report infant birth weight (thus, infant weight-for-age z-score at birth could not be calculated) and one participant did not report her height 
(thus, pre-pregnancy body mass index [BMI] could not be calculated). Given the lack of previous data on this topic, we aimed to recruit a sample size of at least 100 based on guidelines from simulation studies (Bujang et al., 2018; Nemes et al., 2009) and the sample sizes of the few published studies available (Mangel et al., 2019; Puapornpong et al., 2013; Vazirinejad et al., 2009). The final sample size was small (Bujang et al., 2018; Nemes et al., 2009), but allowed for preliminary descriptive analyses that can serve as a foundation for future research.

\section{Measurement}

Variability in Breast Anatomy. Nipple and breast parameters of interest included nipple base width, nipple length, and areolar density assessed on the right and left breast separately (Figure 1). Measures of variability in breast anatomy were adapted from Wilson-Clay and Hoover's (2017) descriptions. All measurements were conducted by an International Board Certified Lactation Consultant (IBCLC) in a temperature-controlled room. Measurements were taken prior to nursing and pumping. Intra-rater reliability for nipple base width, nipple length, and areola density measures was established prior to data collection and was high $(\rho>.90)$ for all measures.

Nipple base width was assessed via a nipple ruler that was specifically designed for this study. The ruler consisted of circular cut-outs that fit around the base of the nipple and assessed nipple base diameter in $1 \mathrm{~mm}$ increments; circular cut-outs ranged from $7 \mathrm{~mm}$ to $40 \mathrm{~mm}$ (Stark, 1994; Wilson-Clay \& Hoover, 2017; Ziemer \& Pigeon, 1993; Ziemer et al., 1995). The nipple base diameter was determined by placing the ruler at the base of the nipple, at the point where the base of the nipple met the areola. The recorded nipple base diameter was the cut-out that allowed the ruler to be placed without rubbing against the sides of the nipple.

Nipple length was assessed with a standard ruler. The end of the ruler was placed at the base of the nipple and the length of the ruler was aligned with the length of the nipple (Wilson-Clay \& Hoover, 2017). Nipple length was then measured in $1 \mathrm{~mm}$ increments. Per the measurement protocol developed by the investigators, inverted nipples would have been measured without any manipulation of the nipple; however, no inverted nipples were encountered.

Areolar density was assessed via manual assessment of areola compressibility at the base of the nipple. To do this, the IBCLC placed her index finger on the top and her thumb on the bottom of the base of the nipple-areolar area and compressed. A scoring system developed for this study was then used to describe the level of compressibility the IBCLC observed. The areolar density was given a score of 1 if there was no pliability when the base of the nippleareolar area was compressed with the forefinger and thumb. A score of 2 indicated that the breast possessed

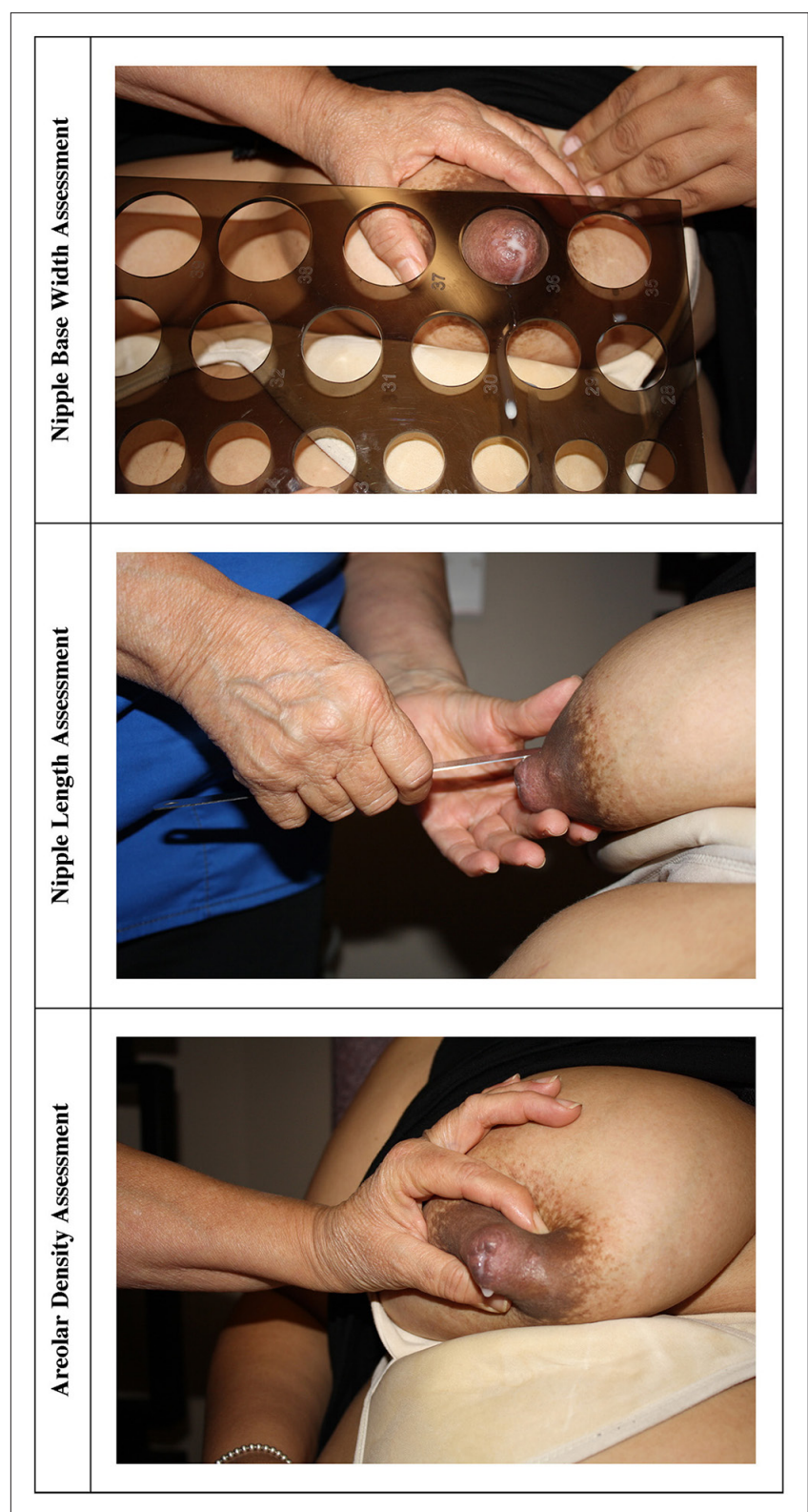

Figure I. Measurement of Variability in Breast Anatomy.

some pliability when the base of the nipple-areolar area was compressed with the forefinger and thumb. A score of 3 was given when there was complete pliability (taking into consideration breast tissue) when the base of the nipple-areolar area was compressed with the forefinger and thumb.

Early Breastfeeding Challenges. The IBCLC assessed whether the participant was currently, or had previously, experienced breastfeeding challenges through a combination of maternal report and clinical assessment. The measures used to assess early breastfeeding challenges were based on standard clinical practices and benchmarks (Flaherman et al., 2015; Huggins, 2020; Kellams et al., 2017; Lauwers \& Swisher, 2016). Each participant 
self-reported her experiences of sore nipples, cracked nipples, or mastitis, and, if currently experienced, the IBCLC confirmed the report.

The participants also self-reported any difficulties with latch, low milk supply, and slow infant weight gain; however, these were only documented when the IBCLC confirmed the information through clinical assessment and based on standard clinical benchmarks (Flaherman et al., 2015; Huggins, 2020; Kellams et al., 2017; Lauwers \& Swisher, 2016). To assess difficulty with latch, the IBCLC first assessed the participant while breastfeeding, including whether the latch appeared ineffective and whether the participant experienced discomfort when the infant was nursing. The IBCLC assessed difficulties with latch, sore nipples, cracked nipples, and mastitis on the left and right breast separately. If the IBCLC assessed the latch to be ineffective or uncomfortable for the participant, the IBCLC then assessed the quality of the infant's positioning and suck, specifically assessing whether the infant was able to achieve suction, deep and rhythmic sucking (versus shallow and fluttering sucking), and audible swallowing.

To assess low milk supply, the IBCLC measured total intake by weighing the infant before and after a complete nursing. The IBCLC measured the residual breastmilk by having the participant apply nipple cream to the base of each nipple and then pumping the milk using a hospitalgrade, bilateral breast pump kit for 10-12 min. The IBCLC then assessed the combined adequacy of the volume of infant intake and residual milk volume within the context of when the participant reported the infant last nursed. This information was then compared to clinical standards for age-specific expected volumes (Huggins, 2020). A participant was classified as having low milk supply if producing below the clinical standards (Huggins, 2020).

To assess slow infant weight gain, the IBCLC weighed the infant using a standard infant scale that had a resolution of $2 \mathrm{~g}$ and an accuracy of $\pm 0.034 \%$ (Medela Baby Weigh $^{\text {TM }}$ II, Medela, LLC, McHenry, IL USA). Participants reported their infants' birth weight, and, when possible, the IBCLC confirmed this from the infant's medical chart. Weight change from birth was calculated and compared to clinical standards (Kellams et al., 2017). An infant was classified as having slow infant weight gain if he/she had not returned to his/her weight by 10-14 days after birth or if the infant was not gaining $\sim 1$ oz per day after Day 5 (Flaherman et al., 2015).

Covariates. The researchers developed a demographics questionnaire that provided the following demographic characteristics: participant age, parity, marital status, education level, family income level, race/ethnicity, and participation in federal assistance programs (e.g., Special Supplemental Nutrition Program for Women, Infants, and Children [WIC], and infant sex, age, and feeding history. With respect to infant feeding history, participants were also asked to report whether they had ever given their infant formula and, if so, their infant's age at the time of complementation. Participants also reported whether or not they were currently pumping.

Participants self-reported their pre-pregnancy weight and height. Weight and height were used to calculate participants' pre-pregnancy Body Mass Index (BMI = weight $[\mathrm{kg}] /$ height $\left.\left[\mathrm{m}^{2}\right]\right)$. Participants with a BMI $<25 \mathrm{~kg} / \mathrm{m}^{2}$ were classified as normal weight and participants with a BMI $\geq 25 \mathrm{~kg} / \mathrm{m}^{2}$ were classified as overweight or obese. Participants also reported their infants' birth weight and length. One participant did not report her infant's birth weight or length, two participants did not report their infants' birth weight, and five participants did not report their infants' birth length. Thus, birth weight was available for 116 infants and birth length was reported for 113 infants. Given more data were available for birth weight than length, birth weight was normalized to z-scores for weight-for-age (WAZ) using the World Health Organization Growth Standards (WHO Multicentre Growth Reference Study Group, 2006). Birth WAZ was used in subsequent descriptive and inferential analyses.

Participants were given the option to complete questionnaires assessing family demographics and their infants' feeding history in the exam room or at home. Participants who opted to complete the questionnaires at home were also given a self-addressed stamped envelope with which to mail the questionnaire packets back. Given that all participants attended the clinic visit with their infants, the majority of participants opted to complete the questionnaires at home at a time when their infant was asleep or with another caregiver. Some participants $(n=23)$ did not complete or return the questionnaires despite repeated contact attempts to request questionnaire completion. To maximize available data, participants who failed to complete the questionnaires or failed to answer select questions on the questionnaires were kept in the sample and were categorized as "Not Reported" for missing demographic and infant feeding characteristics. A copy of all data collection tools and questionnaires used in this study are available in Appendix 1, available online.

\section{Data Collection}

We conducted this study between April 2018 and April 2019. All mothers who visited the FHMC Breastfeeding Center and who met the eligibility criteria were invited to participate in the study. During this invitation, the study purpose was explained, the voluntary nature of participation was emphasized, and assurance that refusal to participate would not affect the health services they received was provided. Mothers who agreed to participate provided written and oral consent for participation.

One of the study investigators (OM), a bilingual Registered Nurse and IBCLC, completed all of the 
assessments. Assessments were conducted during a single visit and within a private exam room at the Breastfeeding Center to protect participants' confidentiality. Personally identifiable information was not recorded on any study documents and all data collected was stored securely within study offices and accessed only by study personnel.

\section{Data Analysis}

Data were cleaned and checked for normality prior to data analysis. Descriptive statistics were calculated to summarize: (1) sample characteristics; (2) variations in breast anatomy (i.e., range of nipple base widths, nipple lengths, and areolar density scores); and (3) prevalence of early breastfeeding challenges for the study sample. Logistic regression with estimation via Generalized Estimating Equations was used to determine whether variations in breast anatomy predicted the probability of early breastfeeding challenges (i.e., latch problems, sore nipples, cracked nipples, mastitis, low milk supply, slow infant weight gain). A strength of this analytical approach is that it can account for correlations among predictor variables (Orelien, n.d.; SAS Institute Inc., 2015). Thus, this approach allows for the inclusion of right and left breast measurements within a single model without the need to collapse right and left breast measurements into a single variable, which could increase risk of information loss.

For logistic regression models predicting risk for early breastfeeding challenges, separate models were fit for each breastfeeding challenge examined (six models total). The following covariates were included in each model: infant age and birth WAZ score, maternal age, pre-pregnancy BMI, and parity. During model fitting for each breastfeeding challenge, possible interactive effects of variations in breast anatomy were considered by first fitting models that included three-way interactions between nipple base width, nipple length, and areolar density, as well as main effects of nipple base width, nipple length, and areolar density. If the three-way interaction was not significant, then a model with two-way interactions was explored. If no two-way interactions were significant, then a model with only main effects was considered the best-fit model for the outcome of interest. Continuous predictors (nipple base width and nipple length) were centered around the mean (cWidth, cLength) before computing interaction terms.

Preliminary analyses revealed that incidence of mastitis was very low (i.e., only two participants reported that they experienced mastitis) and logistic regression models did not converge given the large imbalance between outcome groups. Thus, findings for models predicting probability of mastitis were not considered reliable and were not reported. A significance level of $p<.05$ was used to indicate significant differences. All data analyses were conducted using SAS v.9.4 (July 2013; SAS Institute Inc., NC, USA).

\section{Results}

\section{Sample Characteristics and Variations in Breast Anatomy}

Sample characteristics are presented in Table 1. The mean age of participants was 30.1 years $(S D=4.4)$. The mean age of infants was 2.0 weeks $(S D=1.2)$. Infant age ranged from 0.4 weeks to 5.4 weeks; $67 \%(n=79)$ of infants were $\leq 2$ weeks of age at assessment. Average infant weight-forage z-score at birth was $0.29(S D=0.94)$.

Nipple base width and nipple length for the sample are summarized in Table 2. The range of nipple base width diameters was $15-34 \mathrm{~mm}$. Left breast and right breast nipple base widths did not significantly differ $(p=.5879)$. The range of nipple lengths was $5-20 \mathrm{~mm}$. The length of right breast nipples was significantly greater than the length of left breast nipples $(p=.0126)$. Approximately $35 \%(n=$ 42) of participants had dense areolas (compression score of 1-2) on the left breast, and $36 \%(n=43)$ of participants had dense areolas on the right breast. The proportion of left breasts with dense areolas did not differ significantly from the proportion of right breasts with dense areolas (paired $t[118]=-0.28, p=.7828)$.

\section{Associations Between Variations in Breast Anatomy and Early Breastfeeding Challenges}

The percentages and numbers of participants who experienced difficulties with latch, sore nipples, cracked nipples, and/or mastitis on the left and right breasts are summarized in Table 3. Low milk supply was diagnosed for $23 \%(n=$ 27) of participants. Slow infant weight gain was diagnosed for $19 \%(n=22)$ of participants.

For latch problems, the three-way interaction between nipple base width, nipple length, and areolar density was not significant $(p=.8897)$, thus the final model only included two-way interactions (Supplemental Table 1). There was a significant interaction between width and length $(p=.0260)$. As illustrated in Figure 2, there was a significant association between greater nipple base width and probability of latch problems for participants with longer nipples $(p=.0212)$. There was no association between nipple base width and probability of latch problems for participants with shorter nipples $(p=.9989)$.

For sore nipples, the three-way interaction between nipple base width, nipple length, and areolar density was not significant $(p=.4259)$, thus the final model only included two-way interactions (Supplemental Table 2). There was a significant interaction between nipple width and areolar density $(p=.0058)$. As illustrated in Figure 3, when 
Table I. Descriptive Characteristics of Study Sample $(n=119)$.

\begin{tabular}{|c|c|}
\hline Characteristic & $n(\%)$ \\
\hline \multicolumn{2}{|l|}{ Mother Characteristics } \\
\hline \multicolumn{2}{|l|}{ Pre-pregnancy weight status ${ }^{\mathrm{a}}$} \\
\hline Normal weight $\left(\mathrm{BMI}<25 \mathrm{~kg} / \mathrm{m}^{2}\right)$ & $73(6 \mid .3)$ \\
\hline Overweight (BMI $25-29.9 \mathrm{~kg} / \mathrm{m}^{2}$ ) & $24(20.1)$ \\
\hline Obese $\left(\mathrm{BMI} \geq 30 \mathrm{~kg} / \mathrm{m}^{2}\right)$ & $21(17.6)$ \\
\hline \multicolumn{2}{|l|}{ Parity $^{b}$} \\
\hline Primiparous & $50(42.0)$ \\
\hline Multiparous & $36(30.3)$ \\
\hline \multicolumn{2}{|l|}{ Married $^{c}$} \\
\hline Married & $72(60.5)$ \\
\hline Not married & $24(20.2)$ \\
\hline \multicolumn{2}{|l|}{ Education Level $^{d}$} \\
\hline Less than a high school degree & $7(5.9)$ \\
\hline High School Degree & $10(8.4)$ \\
\hline Some college & $20(16.8)$ \\
\hline College graduate & $57(47.9)$ \\
\hline \multicolumn{2}{|l|}{ Family Income Level ${ }^{\mathrm{c}}$} \\
\hline Under $\$ 25,000$ & $16(13.5)$ \\
\hline$\$ 25,000-\$ 75,000$ & $31(26.1)$ \\
\hline Over $\$ 75,000$ & $49(4 \mid .2)$ \\
\hline \multicolumn{2}{|l|}{ Race/Ethnicity ${ }^{\mathrm{e}}$} \\
\hline Non-Hispanic/Latino/Latinx & $54(45.4)$ \\
\hline Hispanic/Latino/Latinx & $33(27.7)$ \\
\hline Other & $8(6.7)$ \\
\hline \multicolumn{2}{|l|}{ Federal Assistance ${ }^{c}$} \\
\hline Participating & $22(18.5)$ \\
\hline Not participating & $74(62.8)$ \\
\hline \multicolumn{2}{|l|}{ Infant Characteristics } \\
\hline \multicolumn{2}{|l|}{ Sex } \\
\hline Female & $59(49.6)$ \\
\hline Male & $60(50.4)$ \\
\hline \multicolumn{2}{|l|}{ Infant Feeding } \\
\hline \multicolumn{2}{|l|}{ Ever Complemented with Formula ${ }^{f}$} \\
\hline Yes & $36(39.1)$ \\
\hline No & $56(47.1)$ \\
\hline \multicolumn{2}{|c|}{ Infant age when complemented with formula ${ }^{g}$} \\
\hline I day or less & $9(9.8)$ \\
\hline $2-6$ days & $25(27.2)$ \\
\hline $7-13$ days & $2(2.1)$ \\
\hline \multicolumn{2}{|l|}{ Currently Pumping ${ }^{f}$} \\
\hline Yes & $70(76.1)$ \\
\hline No & $22(23.9)$ \\
\hline
\end{tabular}

Note. ${ }^{a}$ Not reported by one participant. ${ }^{b}$ Not reported by 33 participants. 'Not reported by 23 participants. ${ }^{d}$ Not reported by 25 participants. ${ }^{e}$ Not reported by 24 participants. ${ }^{f}$ Not reported by 27 participants. ${ }^{g} n=36$.

participants had more dense areolas (compression score of 1 or 2), there was a positive association between nipple width and probability of sore nipples $(p=.0116)$. When participants had less dense areolas (compression score of
$3)$, there was no association between nipple base width and probability of sore nipples $(p=.3388)$.

For cracked nipples, none of the models were predictive (Supplemental Table 3). The three-way interaction between nipple base width, nipple length, and areolar density was not significant $(p=.7352)$. Non-significant two-way interactions were noted between nipple base width and nipple length $(p=.3175)$, nipple length and areolar density $(p=.2772)$, and nipple base width and areolar density $(p=.2880)$. Nipple base width $(p=$ $.4022)$, nipple length $(p=.9499)$, and areolar density ( $p$ $=.2849$ ) were not significant predictors of participants' probability of experiencing cracked nipples.

For low milk supply, the three-way interaction between nipple base width, nipple length, and areolar density was not significant $(p=.3309)$, thus the final model only included two-way interactions (Supplemental Table 4). There was a significant interaction between nipple length and areolar density $(p=.0345)$. As illustrated in Figure 4, when participants had more dense areolas (compression score of 1 or 2), there was no association between nipple length and probability of low milk supply $(p=.2582)$. When participants had less dense areolas (compression score of 3 ), there was a positive association between nipple length and probability of low milk supply $(p=.0474)$.

For slow infant weight gain, the three-way interaction between width, length, and compression was significant $(p=.0006$; Supplemental Table 5). As illustrated in Figure 5, at higher areola densities (compression score of 1 or 2), the combination of shorter and wider nipples was associated with greater probability of slow infant weight gain $(p<.0001$; Figure 5, Panel A). At lower areola density (compression score of 3), the interaction between nipple base width and length were not associated with probability of slow infant weight gain $(p>.05$; Figure 5, Panel B).

\section{Discussion}

Clinical observations suggest variations in breast anatomy, defined as between-participant differences in the width and length of nipples and the density of areolas, may influence some mother-infant dyads' abilities to effectively establish breastfeeding during early postpartum. However, to date, few studies have been available to provide empirical evidence related to whether and how variations in breast anatomy may relate to common early breastfeeding challenges. The present study was a first step toward addressing this research gap. A strength of our approach is that we did not look at breast parameters individually; rather we adopted a holistic approach wherein we considered the parameters together. This approach allowed for identification of the combinations of breast parameters that were predictive of early breastfeeding challenges. 
Table 2. Descriptive Statistics for Nipple Base Width and Nipple Length $(n=119)$.

\begin{tabular}{|c|c|c|c|c|}
\hline & Left Breast & Right Breast & & \\
\hline Measurement (in mm) & $M(S D)$ & $M(S D)$ & $t$ & $p$ \\
\hline Nipple base width & $23.4(3.0)$ & $23.5(3.1)$ & -0.54 & .5879 \\
\hline Nipple length & $8.5(3.2)$ & $9.1(3.2)$ & -2.53 & .0126 \\
\hline
\end{tabular}

Note. Diameter assessed in I mm increments via a nipple ruler consisting of circular cut-outs, ranging from 7-40 mm, that fit around the base of the nipple. Length assessed in I mm increments via a standard ruler.

There are several possible explanations, not mutually exclusive, for why variations in the width and length of nipples and density of areolas were associated with challenges related to latch, nipple soreness, milk supply, and/or infant weight gain. First, it is possible that a mismatch between the large size of a mother's nipple and the small size of an infant's oral cavity could interfere with the infant's ability to establish an appropriate latch (Wilson-Clay \& Hoover, 2017). The relative size of the nipple and density of the areola may make it difficult for the infant to take the right depth of breast tissue into his or her oral cavity or may overwhelm the oral cavity, hindering the coordinated movements of the jaw, tongue, and soft palate (Douglas \& Geddes, 2018; Geddes \& Sakalidis, 2016). In addition, wider nipples and denser areolas may be more difficult for young infants to compress (Alatalo et al., 2019) or may make it more difficult for the infant to latch onto the breast, leading to nipple soreness (Puapornpong et al., 2017). Although these issues would likely resolve as the infant grows and matures, it is quite possible that an inappropriate latch could cause the infant to develop ineffective sucking habits or lead to poor milk transfer and other breastfeeding challenges, resulting in breastfeeding cessation prior to the point when a better match between maternal and infant anatomy is achieved.

With respect to low milk supply and slow infant weight gain, low tissue pliability combined with shorter, wider nipples may make it difficult for young infants to adequately latch onto the breast, negatively affecting the first step in the cascade of events necessary for

Table 3. Prevalence of Early Breastfeeding Challenges $(n=119)$.

\begin{tabular}{lccc}
\hline & Left Breast & & Right Breast \\
\cline { 2 - 2 } Breastfeeding Challenge & $n(\%)$ & & $n(\%)$ \\
\hline Difficulties with latch & $96(80.7)$ & & $94(79.0)$ \\
Sore nipple & $97(81.5)$ & & $95(79.8)$ \\
Cracked nipple & $47(39.5)$ & & $46(38.7)$ \\
Mastitis & $2(1.7)$ & & I (0.84) \\
\hline
\end{tabular}

Note. Early breastfeeding challenges were assessed via a combination of maternal report and clinical assessment by a trained investigator who is a Registered Nurse and International Board Certified Lactation Consultant (IBCLC). successful transfer of milk (Douglas \& Geddes, 2018). In contrast, when breast tissue is more pliable, longer and wider nipples may result in too much breast tissue within the infants' oral cavity, thus inhibiting the intraoral nipple placement, tongue movements, and nipple elongation needed to elicit milk ejection (Sakalidis \& Geddes, 2016). Thus, it is possible both cases may lead to low milk transfer resulting in both lower milk production and slower infant weight gain. Future research should employ longitudinal methods to better understand the potential impact of variations in breast anatomy on breastfeeding experiences and outcomes and how variations in breast anatomy change across the postpartum period. It is also imperative that future research better consider what the infant "brings to the table" and whether factors (e.g., lower birth weight or impaired suck) interact with variations in breast anatomy to heighten risk for early breastfeeding challenges.

Given the paucity of studies examining variations in breast anatomy, the present report of the range of nipple base widths, nipple lengths, and areolar densities provides valuable reference data for future research and practice. The range and distribution of nipple base widths noted is similar to the limited published data available; however, the sample average within the present study is somewhat higher (Stark, 1994; Wilson-Clay \& Hoover, 2017; Ziemer \& Pigeon, 1993; Ziemer et al., 1995). For example, Zeimer and colleagues reported average nipple diameters ranging from $15 \mathrm{~mm}$ to $16 \mathrm{~mm}$ (Ziemer \& Pigeon, 1993; Ziemer et al., 1995). WilsonClay and Hoover (2017) noted clinical observations of nipple diameter in samples of 34 and 100 women who sought assistance from IBCLCs for breastfeeding challenges. Within these samples, $70 \%$ and $58 \%$ of women, respectively, had nipples diameters ranging from $16 \mathrm{~mm}$ to $23 \mathrm{~mm}$. In addition, in the sample of 100 women, average nipple diameter was $17.5 \mathrm{~mm}$ (Wilson-Clay \& Hoover, 2017). Recently, Mangel et al. (2019) reported average nipple diameters of $14.7(S D=4.4) \mathrm{mm}, 16.3$ $(S D=3.7) \mathrm{mm}, 18.0(S D=8.7) \mathrm{mm}$, and $15.7(S D=2.9)$ $\mathrm{mm}$ in women assessed as underweight, normal weight, overweight, and obese, respectively. However, it is important to note that within all of these sources, precise details about how nipple diameter was measured were 


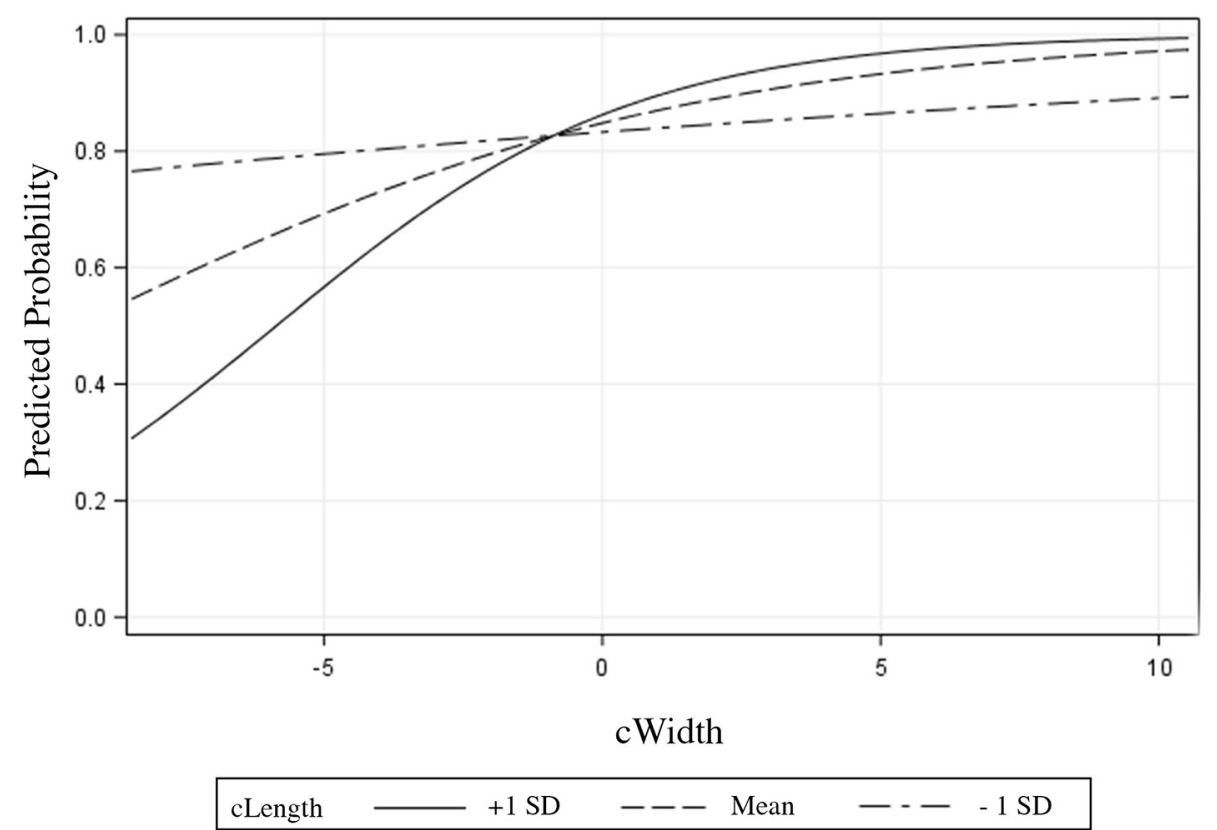

Figure 2. Predicted Probability of Latch Problems: Interaction Between Nipple Base Width and Nipple Length $(n=1$ I5).

not provided (e.g., at the base of the nipple versus at the tip) and measurement techniques varied (e.g., standard rulers, calipers, engineers' circle template). In addition, our range of nipple base measurements align with the width of available products (e.g., nipple shields and flanges) that are fitted to the width of the nipple base (e.g., Medela, n.d.). Thus, it is possible that these discrepancies are due to inconsistency in the location and method of the measurement across these studies and clinical observations. With regard to nipple length, our values are consistent with those of Wilson-Clay and Hoover (2017), who reported an average nipple length of $9.5 \mathrm{~mm}$ in a clinical sample of 100 women. Further research within a larger, more representative sample of

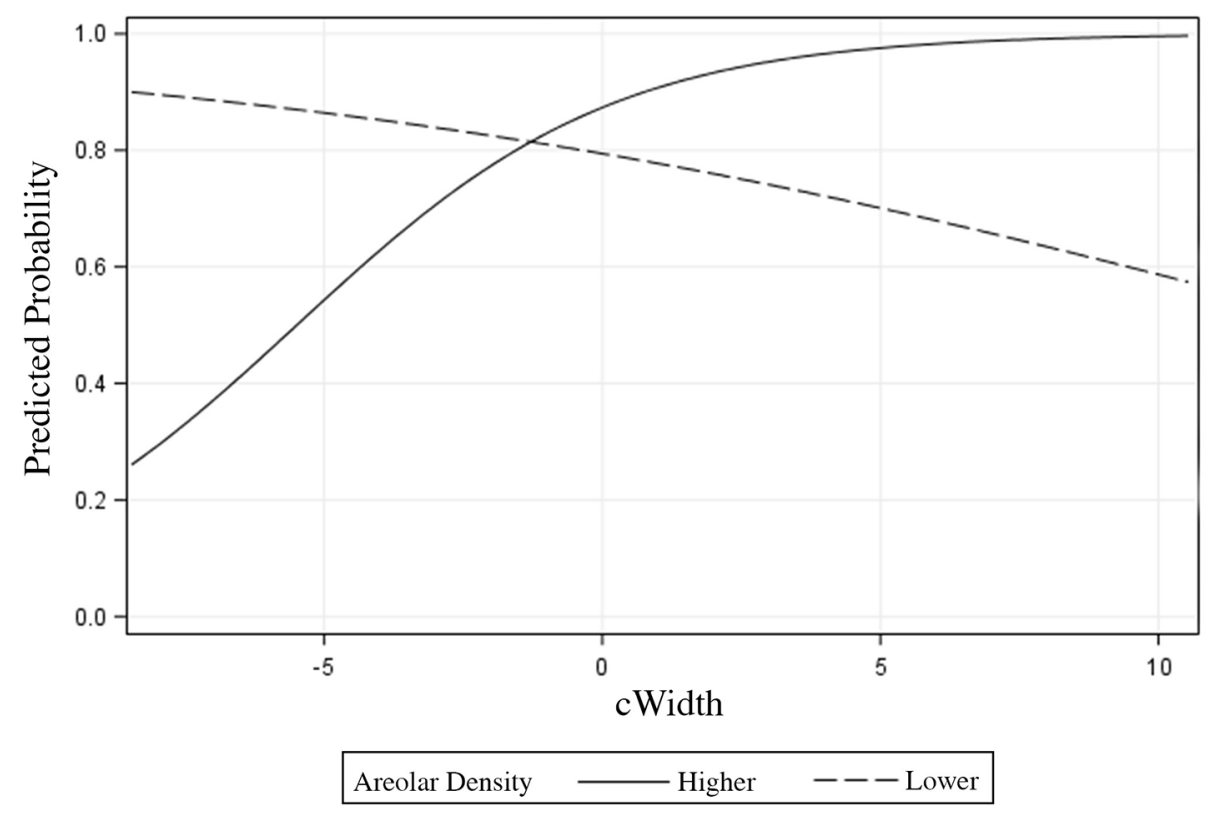

Figure 3. Predicted Probability of Sore Nipples: Interaction Between Nipple Base Width and Areolar Density $(n=1$ I5). 


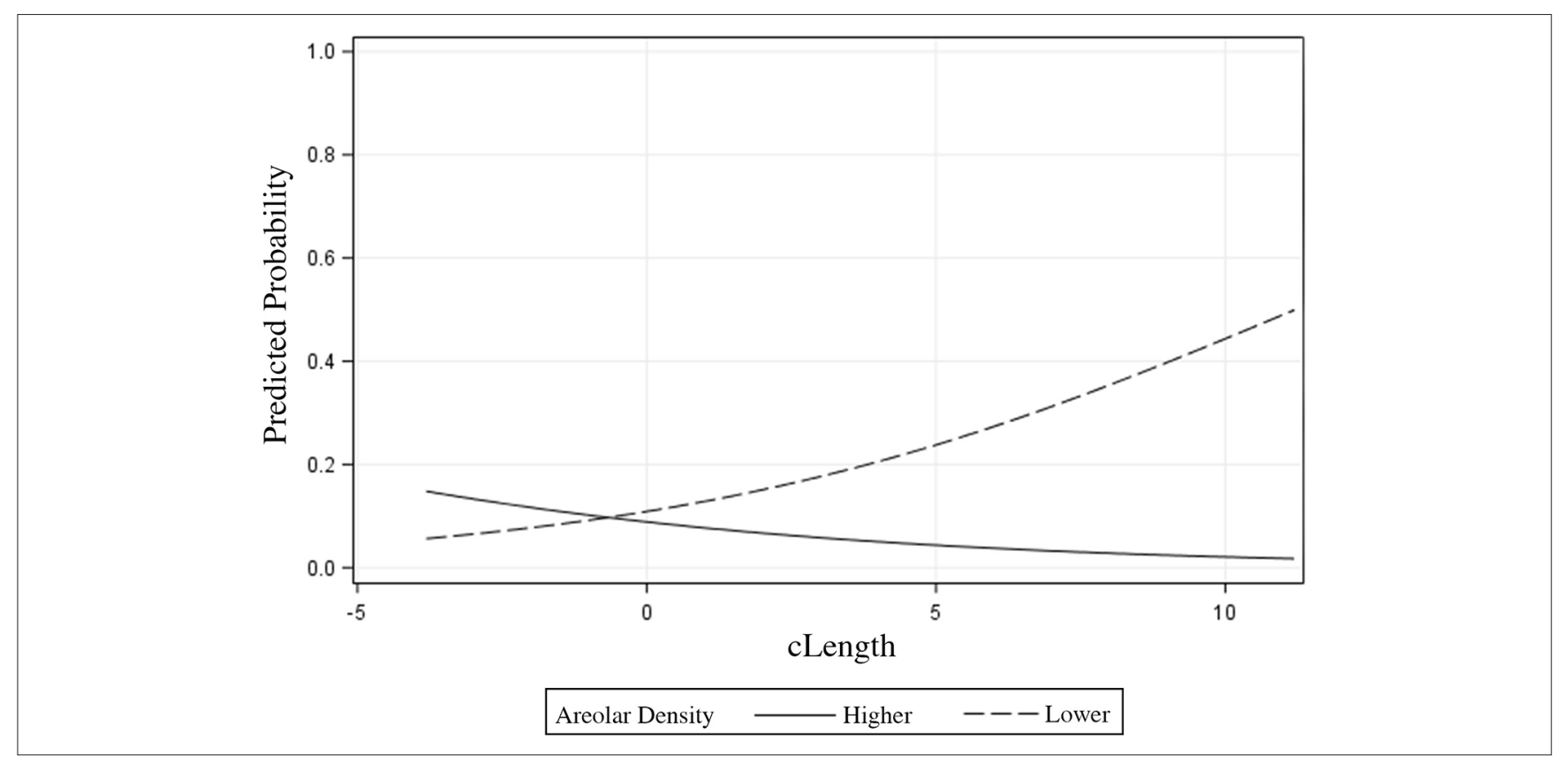

Figure 4. Predicted Probability of Sore Nipples: Interaction Between Nipple Base Width and Areolar Density $(n=1 \mathrm{I} 5)$.

mothers and infants is warranted to better understand differences between our data and previously published measurements. This research could work to increase the precision of the measures developed for the present study and could also explore the relevance of additional measures of breast anatomy, including the nipple tip face, intermammary space, or asymmetry of the breasts.

\section{Limitations}

It is important to acknowledge that the present study was cross-sectional and observational; thus, our interpretations are only speculations about possible explanations for why certain variations in breast anatomy were associated with early breastfeeding challenges. We focused on only three aspects of breast anatomy; thus, our perspective may have been limited because we did not measure other breast parameters. We also did not consider how infant characteristics, including lower birth weight or impaired suck, interacted with variations in breast anatomy to heighten risk for early breastfeeding challenges. Further, a minority of participants were assessed between 2 and 6 weeks postpartum versus the first 2 weeks postpartum. It is possible that nipple base width and length and areolar density changed across the first 6 weeks postpartum and that our measures may have been biased based on when the participant was assessed.

Our study sample included primarily non-Hispanic/ Latino/Latinx white (45\%) and Hispanic/Latino/Latinx (28\%) participants, limiting the generalization of our findings to the broader population. In addition, this study was conducted in one breastfeeding clinic where mothers self-selected whether or not to visit the clinic, and one IBCLC assessed all participants. This limited scope may have biased our sample and may limit the generalizability of our findings. A superior approach would be to have multiple IBCLCs assess participants with demonstrated inter-rater reliability to improve the validity and reliability of our key study assessments.

\section{Conclusions}

In conclusion, the benefits of breastfeeding are widely known and appreciated, yet significant proportions of the population still fail to meet breastfeeding recommendations and goals. Much previous research has elucidated psychological and sociocultural barriers to breastfeeding, while relatively few investigators have explored whether tailored support should be provided to mothers based on their individual variations in breast anatomy. Findings from the present study provide support for further research examining short- and longterm outcomes. Findings also support further clinical research exploring possible forms of counseling or interventions that could be tailored to help mothers understand their specific variations in breast anatomy and how to maximize their potential for breastfeeding success. 


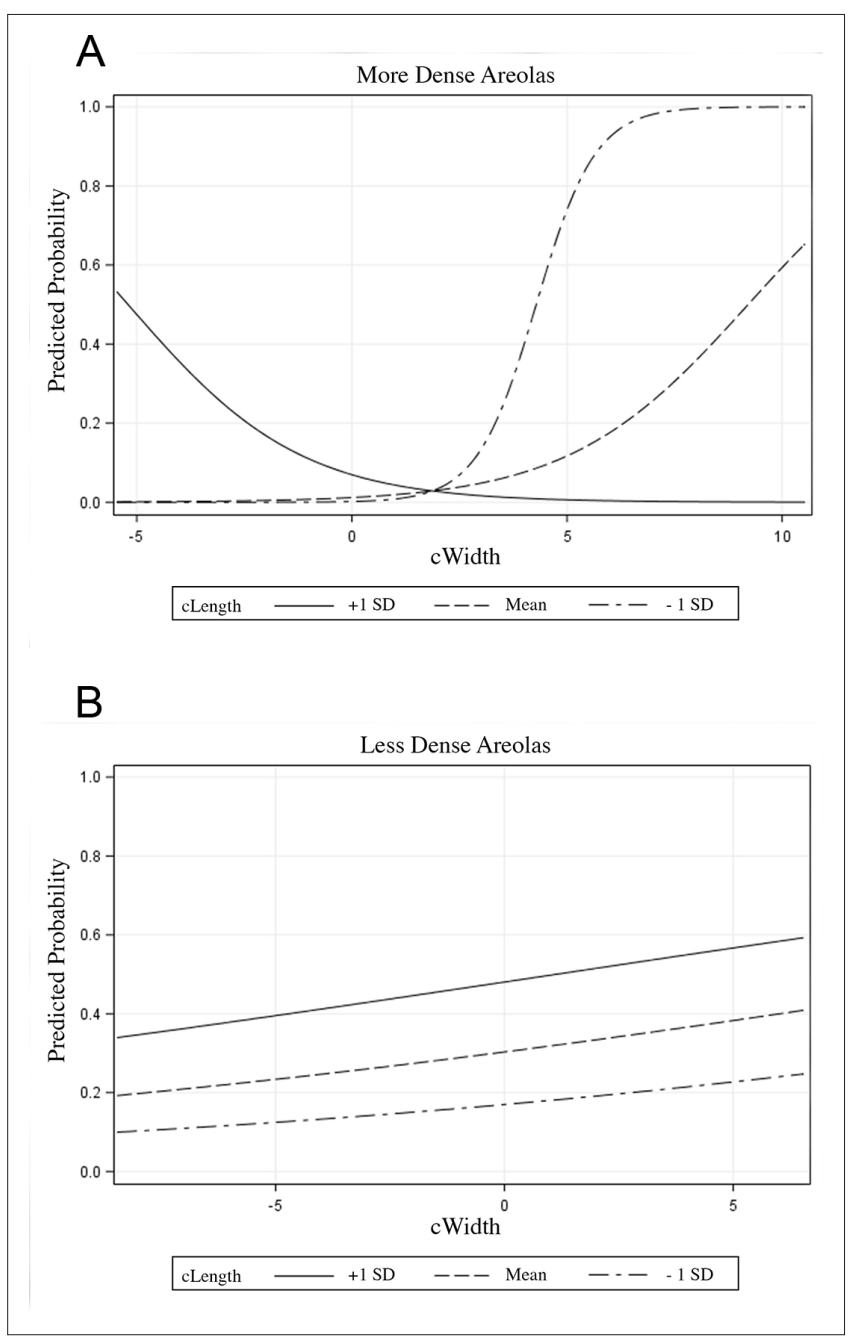

Figure 5. Panel A. Predicted Probability of Slow Infant Weight Gain at Higher Areolar Densities: Three-way Interaction Between Nipple Base Width, Nipple Length, and Areolar Density $(n=1$ I5). Panel B. Predicted Probability of Slow Infant Weight Gain at Lower Areolar Density: Three-way Interaction Between Nipple Base Width, Nipple Length, and Areolar Density $(n=115)$.

\section{Acknowledgments}

We thank the mothers and infants who participated in this study. This project was supported by a Cal Poly Center for Health Research Seed Grant. The authors have no conflicts of interest relevant to this article to disclose.

\section{Declaration of Conflicting Interests}

The authors have no conflicts of interest relevant to this article to disclose.

\section{Funding}

This project was supported by a California Polytechnic State University Center for Health Research Seed Grant.

\section{ORCID iD}

Alison K. Ventura, PhD, CLEC (D) https://orcid.org/0000-00022948-4446

\section{Supplemental Material}

Supplemental material for this article is available online.

\section{References}

Alatalo, D., Jiang, L., Geddes, D., \& Hassanipour, D. F. (2019). Capturing nipple deformation and peripheral pressure on the areola during breastfeeding. Journal of Biomedical Engineering. Advance online publication. doi:10.1115/1.4043665

American Academy of Pediatrics(2012). Breastfeeding and the use of human milk. Pediatrics, 129(3), e827-e841. doi:10.1542/peds. 2011-3552

Bordens, K., \& Abbot, B. B. (2018). Research design and methods: A process approach (10th ed.). McGraw Hill.

Bujang, M. A., Sa'at, N., Sidik, T., \& Joo, L. C. (2018). Sample size guidelines for logistic regression from observational studies with large population: Emphasis on the accuracy between statistics and parameters based on real life clinical data. Malaysian Journal of Medical Sciences, 25(4), 122-130. doi:10.21315/mjms2018.25.4. 12

Douglas, P., \& Geddes, D. (2018). Practice-based interpretation of ultrasound studies leads the way to more effective clinical support and less pharmaceutical and surgical intervention for breastfeeding infants. Midwifery, 58, 145-155. doi:10.1016/j.midw.2017.12.007

Feenstra, M. M., Jørgine Kirkeby, M., Thygesen, M., Danbjørg, D. B., \& Kronborg, H. (2018). Early breastfeeding problems: A mixed method study of mothers' experiences. Sexual \& Reproductive Healthcare, 16, 167-174. doi:10.1016/j.srhc.2018.04.003

Flaherman, V. J., Schaefer, E. W., Kuzniewicz, M. W., Li, S. X., Walsh, E. M., \& Paul, I. M. (2015). Early weight loss nomograms for exclusively breastfed newborns. Pediatrics, 135(1), e16-e23. doi:10.1542/peds.2014-1532

Geddes, D. T., \& Sakalidis, V. S. (2016). Ultrasound imaging of breastfeeding - A window to the inside: Methodology, normal appearances, and application. Journal of Human Lactation, 32(2), 340-349. doi:10.1177/0890334415626152

Huggins, K. (2020). The nursing mother's companion (8th ed.). The Harvard Common Press.

Kellams, A., Harrel, C., Omage, S., Gregory, C., \& RosenCarole, C. (2017). ABM Clinical Protocol \#3: Supplementary feedings in the healthy term breastfed neonate, Revised 2017. Breastfeeding Medicine, 12(3), 188-198. doi:10.1089/bfm.2017. 29038.ajk

Lauwers, J., \& Swisher, A. (2016). Counseling the nursing mother (6th ed.). Jones \& Bartlett Learning.

Mangel, L., Mimouni, F. B., Mandel, D., Mordechaev, N., \& Marom, R. (2019). Breastfeeding difficulties, breastfeeding duration, maternal Body Mass Index, and breast anatomy: Are they related? Breastfeeding Medicine, 14(5), 342-346. doi:10.1089/bfm.2018. 0262 
Medela. (n.d.). Choosing the right breast shield. https://www.mede labreastfeedingus.com/tips-and-solutions/13/choosing-a-correctlyfitted-breastshield.

Nemes, S., Jonasson, J. M., Genell, A., \& Steineck, G. (2009). Bias in odds ratios by logistic regression modelling and sample size. $B M C$ Medical Research Methodology, 9, 56. doi:10.1186/1471-2288-956

Orelien, J. G. (n.d.). Model fitting in PROC GENMOD. https://support. sas.com/resources/papers/proceedings/proceedings/sugi26/p26426.pdf

Puapornpong, P., Paritakul, P., Suksamarnwong, M., Srisuwan, S., \& Ketsuwan, S. (2017). Nipple pain incidence, the predisposing factors, the recovery period after care management, and the exclusive breastfeeding outcome. Breastfeeding Medicine, 12(3), 169-173. doi:10.1089/bfm.2016.0194

Puapornpong, P., Raungrongmorakot, K., Paritakul, P., Ketsuwan, S., \& Wongin, S. (2013). Nipple length and its relation to success in breastfeeding. Journal of the Medical Association of Thailand, 96(Suppl. 1), S1-S4.

Sakalidis, V. S., \& Geddes, D. T. (2016). Suck-swallow-breathe dynamics in breastfed infants. Journal of Human Lactation, 32(2), 201-211; quiz 393-205. doi:10.1177/0890334415601093

SAS Institute Inc. (2015). The MIXED procedure. In SAS/STAT® 14.1 User's Guide. SAS Institute Inc.
Stark, Y. (1994). Human nipples: Function and anatomical variations in relationship to breastfeeding. (Unpublished master's thesis), Pacific Oaks College, Pasadena, CA.

Tabachnick, B. G., \& Fidell, L. S. (2019). Using multivariate statistics (7th ed.). Pearson.

Vazirinejad, R., Darakhshan, S., Esmaeili, A., \& Hadadian, S. (2009). The effect of maternal breast variations on neonatal weight gain in the first seven days of life. International Breastfeeding Journal, 4, 13. doi:10.1186/1746-4358-4-13

WHO Multicentre Growth Reference Study Group (2006). WHO child growth standards based on length/height, weight, and age. Acta Paediatrica Supplement, 450, 76-85.

Wilson-Clay, B., \& Hoover, K. (2017). The breastfeeding atlas (6th ed.). LactNews Press.

Ziemer, M. M., Cooper, D. M., \& Pigeon, J. G. (1995). Evaluation of a dressing to reduce nipple pain and improve nipple skin condition in breast-feeding women. Nursing Research, 44(6), 347-351. doi:10. 1097/00006199-199511000-00005

Ziemer, M. M., \& Pigeon, J. G. (1993). Skin changes and pain in the nipple during the 1st week of lactation. Journal of Obstetric, Gynecologic \& Neonatal Nursing, 22(3), 247-256. doi:10.1111/j. 1552-6909.1993.tb01806.x 